\title{
Archéopages
}

Archéopages

Archéologie et société

Hors-série 2 | 2010

Archéologie sans frontières

\section{Le savoir-faire des topographes de l'Inrap dans les missions archéologiques françaises à l'étranger}

Patrick Deleuze

\section{(2) OpenEdition}

1 Journals

Édition électronique

URL : https://journals.openedition.org/archeopages/795

DOI : 10.4000/archeopages.795

ISSN : 2269-9872

Éditeur

INRAP - Institut national de recherches archéologiques préventives

Édition imprimée

Date de publication : 1 octobre 2010

Pagination : 93-94

ISSN : $1622-8545$

\section{Référence électronique}

Patrick Deleuze, « Le savoir-faire des topographes de l'Inrap dans les missions archéologiques

françaises à l'étranger », Archéopages [En ligne], Hors-série 2 | 2010, mis en ligne le 01 octobre 2010 consulté le 23 février 2023. URL : http://journals.openedition.org/archeopages/795 ; DOI : https:// doi.org/10.4000/archeopages.795 
les sondages sur les berges des grands trapeang permettront d'aborder la question de l'habitat de manière globale, en menant une réflexion sur l'organisation, le déplacement et l'extension de celui-ci autour des différents sanctuaires. Le projet vise une approche globale, considérant le temple et le village à l'intérieur d'un territoire, lui-même influencé par des limites préexistantes et contraignantes.

BÂTy P., Bolle A., novembre 2005, "Sanctuaires et habitats sous l'aéroport de Siem Reap, Cambodge », Archéologia, n² 427, p. 18-23.

BÂTy P. et al, 2005, «Extension de l'aéroport de Siem Reap, Trapeang Thlok Prasat Trapeang Ropou », Rapport de diagnostic archéologique ; "Trapeang Thlok sanctuaire et habitat », Rapport de fouille, DFS de sauvetage urgent, base Inrap de Poitiers, 1 volume $\mathrm{A}_{3}$ 167 pages, 157 fig., 248 photographies.

BÂTy P., 2007 « Les couteaux angkoriens de Trapeang Thlok et de Prasa Trapeang Ropou », Befeo, 94, p. 95-110.

COEDES G., 1952, Inscriptions du Cambodge, vol. 4, p. 151, Paris, EFEO.

Dagens B., 1994 «Le temple indien en Asie du Sud-Est. Archéologie d'une forme », in BIzOT F. (DIR.), Recherches nouvelles sur le Cambodge, Paris, Efeo, p. 259-272.

GAUCHER J., FrAniatTe M., 1999, Recherches archéologiques à l'aéroport de Siem Reap. Opération Préventive, DFS, Mission archéologique française à Angkor, $157 \mathrm{p}$.

Groslier B. PH., 1979, « La cité hydraulique angkorienne : exploitation ou surexploitation du sol ?», Befeo, 68, p. 161-202.

Groslier B. PH., 1974 « Agriculture et religion dans l'Empire angkorien ", Études Rurales, 53-54-55-56, p. 95-117.

LUNet De LAJONQUiere É. E., 1911, Inventaire descriptif des monuments $d u$ Cambodge, t. III, Efeo, Paris, p. 132

MARCHAL H., 1922 à 1936, Journaux de Fouille - région d'Angkor Archives de l'École française d'extrême orient, Paris.

Marchal H., 1920, Rapport de la conservation d'Angkor, Archives de l'Efeo, Paris.

Pottier Chr., 1999, Carte archéologique de la région d'Angkor zone sud, thèse de doctorat, Université de Paris III, Ufr Orient et Monde Arabe, 3 volumes, 382 p., 33 cartes.

Pottier Chr., 200o, «À la recherche de Goloupura », Befeo, 87, p. 79-107

Trouve G., 1933, Chronique Efeo 1933, Befeo, 33 (2), 1134 p.

\section{Le partenariat entre l'Inrap et l'Autorité pour la protection du Site et de l'Aménagement de la Région d'Angkor}

Ros Borath

Président de la commission nationale pour le Patrimoine mondial, directeur général adjoint de l'APSARA

U n partenariat entre l'Inrap et l'Apsara (Autorité pour la protection du site et l'aménagement de la région d'Angkor) a été initié en 2004 afin de permettre la première opération archéologique préventive de grande ampleur au Cambodge. Cette opération était financée par la Société concessionnaire de l'Aéroport de Siem Reap (filiale du groupe Vinci), aménageur du site de l'aéroport. De fait, le développement du tourisme entraîne des aménagements importants qu'il est nécessaire d'adapter au mieux sans atteindre à l'intégrité de ce patrimoine archéologique et architectural unique. À Angkor, le besoin d'étendre la capacité d'accueil de l'aéroport par la construction d'un nouveau terminal a été reconnu d'utilité publique. Cet important complexe aéroportuaire, existant avant l'inscription du site d'Angkor sur la liste du Patrimoine mondial de l'Unesco (décembre 1992), se retrouve aujourd'hui situé dans la zone $\mathrm{n}^{\circ} 1$, dite de sites monumentaux, méritant le plus haut degré de protection.
L'opération de diagnostic s'est déroulée de janvier à mai 2004 sur une superficie totale de 26 ha. Cette opération s'étant révélée très positive, l'Apsara a prescrit deux opérations de fouilles de sauvetage, de mai à juillet 2004, confiées à un agent de l'Inrap en collaboration avec des archéologues cambodgiens appartenant au département des Monuments et de l'Archéologie et des archéologues intégrés à l'unité d'Archéologie Préventive à la suite de cette expérience (cf. p. 86 article de Pierre Bâty). Ces fouilles ont concerné le temple de Trapeang Thlok, daté du XI ${ }^{\mathrm{e}}$ siècle et un habitat associé. Par ailleurs, l'importance des vestiges révélés par le diagnostic aux abords du temple de Prasat Trapeang Ropou a permis la prise de mesures conservatoires et le gel des terrains concernés, ainsi qu'une étude préliminaire pour la mise en valeur du site par l'unité de Gestion des abords. Les résultats de cette première fouille extensive et de ce premier diagnostic sont importants car ils permettent d'aborder un site dans toute sa complexité : un temple, un village, un espace foncier.

Outre la mise en ouvre et la réalisation de ces opérations, l'un des objectifs a été la formation des archéologues cambodgiens aux méthodes de l'archéologie préventive de grands travaux. La réussite de cette première opération a conduit nos deux établissements publics à se rapprocher par la signature d'une convention cadre de coopération internationale, d'une durée de trois ans, conformément à la politique de transfert de compétences soutenue par la France ainsi que par la Communauté internationale afin de conforter les efforts de l'Apsara.

\section{Le savoir-faire des topographes de l'Inrap dans les missions archéologiques françaises à l'étranger}

Patrick Deleuze

Inrap

$\mathrm{D}$ epuis la création de l'Afan, certains archéologues ou spécialistes associés, parmi lesquels des topographes, se sont engagés individuellement dans des missions à l'étranger, au gré des opportunités et de leurs travaux personnels. Étant moi-même topographe et ayant travaillé à l'Ifao, en Égypte, j'ai été chargé par le président et par le directeur général d'alors (Philippe Cuvillier et Alain Loiseau) d'officialiser et de cadrer une collaboration qui s'était installée de fait. À la création de l'Inrap, le président, Jean-Paul Demoule, et la directrice générale, Nicole Pot, ont intégré ces missions dans le cadre des projets d'actions scientifiques à l'étranger, sous la responsabilité de Nathan Schlanger.

À partir de 1997, sous la forme de prestations, plusieurs collègues de l'Afan ont participé à des activités de coopération culturelle qui s'inscrivaient dans l'esprit de partenariat nord-sud que la 
direction entendait développer, dans le cadre des missions archéologiques françaises à l'étranger soutenues par le ministère des Affaires étrangères.

Les topographes furent concernés au premier chef par ces demandes de collaboration. Citons, à titre d'exemple, pour l'année 1998, le relevé de la nécropole d'El Deir, à l'ouest de Louqsor, par Xavier Chadefaux (direction F. Dunand, université Marc Bloch, Strasbourg), celui du site urbain de Dia, au Mali, par Pierre Texier (direction Rogier Bedaux, musée de Leiden, Pays-Bas) et le début d'une collaboration suivie, intégrant également d'autres spécialistes de l'Inrap, avec le Centre français des études éthiopiennes (cf. p. WWW). Depuis, ces missions se sont rapidement multipliées, notamment en Afrique et en Asie, et chaque année, plusieurs topographes de l'Inrap contribuent à l'avancement de l'archéologie française à l'étranger : en 1999, Pierrick Leblanc a effectué le relevé du temple romain de Yanouh, au Liban, à la demande de la Maison de l'Orient méditerranéen ; en 2004, sur le site prédynastique de Kom el-Khilgan (cf. p. XXXX), dans le delta du Nil, Bruno Fabry a relevé l'ensemble du tell et de la nécropole (direction Béatrix Midant-Reynes, CNRS); depuis 2007, Pascal Raymond a fait de la topographie une des disciplines pivot de la mission Mentesh-Tépé en Azerbaïdjan (direction Bertille Lyonnet, CNRS) (cf. p. 73).

L'enregistrement de structures archéologiques vouées à la destruction exige, en effet, un grand professionnalisme permettant de les restituer avec précision. L'implantation des maillages et les plans topographiques de surfaces souvent supérieures à plusieurs hectares sont délicats à réaliser. Ces tâches ont été assurées, à l'Afan comme à l'Inrap, par des topographes qui ont su constituer et renouveler un éventail d'outils et de logiciels de pointe qui servent autant de soutien à l'élaboration des hypothèses et des problématiques de recherche que d'aide à la gestion pratique du chantier.

Aujourd'hui, les topographes de l'Inrap utilisent, pour les relevés, des tachéomètres laser et des GPS permettant une prise de points avec une précision inférieure à $10 \mathrm{~cm}$; pour le dessin, AutocadCovadis, ainsi que la géo-codification et le recours au tracé automatique ; pour la restitution, Photoplan, logiciel de redressement des photos de vestiges en fonction des cibles topographiques et 3DS Max, pour les vues en trois dimensions. La somme de ces données permet, en outre, de nourrir les fonds à partir desquels s'élaborent les SIG. Le choix de ces outils permet aux topographes de l'Inrap d'allier la qualité dans la précision à la rapidité dans l'acquisition des points de relevés et leurs traitements graphiques. Les résultats sont tellement pertinents qu'il semblerait insensé de réaliser une opération de fouille ou de diagnostic sans cet appui topographique. C'est cette qualité et cette maîtrise que les organismes partenaires apprécient. Ils reconnaissent également volontiers que les compétences des topographes de l'Inrap ont souvent contribué à faire évoluer non seulement leurs propres techniques mais aussi leur appréhension des sites, comme le précisait
Jean Guilaine, professeur au Collège de France et directeur de la mission sur le site de Shillourokambos, à Chypre, après sa collaboration avec Frédéric Boumier, de1999 à 2002, suivie de celle avec Vincent Pommier, en 2003 et 2004.

\section{Participation de l'Inrap aux programmes de sauvegarde du site d'Angkor}

Éric Llopis
Inrap

$\mathrm{D}$ e 2003 à 2004, puis de 2006 à 2009, l'Inrap a participé aux programmes soutenus par l'ambassade de France au Cambodge pour la protection du patrimoine et le développement du site d'Angkor. L'objectif de la mission était principalement d'apporter un appui institutionnel et des savoir-faire optimaux par des actions d'ordre divers : l'établissement de cahiers des charges et de programmations des opérations d'archéologie préventive liées aux projets d'aménagement élaborés par l'Apsara (Autorité pour la protection du site et l'aménagement de la région d'Angkor); l'encadrement technique et scientifique des interventions préventives menées par les divers départements de l'Apsara; la définition des potentiels archéologiques, afin de constituer un outil de gestion des aménagements et contribuer à une meilleure connaissance des sites; la formation du personnel archéologique aux méthodes d'intervention et d'enregistrement ; la production de documents divers réservés à la constitution des dossiers de présentation : projets, CIC et autres manifestations, ainsi que la proposition de programmes de recherche pour la réalisation de « chantiers-écoles » ; l'émission de cahiers des charges et de programmation de l'opération d'archéologie préventive sur le site de Preah Vihear, dans le cadre d'un projet global de sauvegarde et de mise en valeur du site, sous l'égide de l'Autorité nationale de Preah Vihear et de l'Unesco; l'intervention traitant de l'archéologie préventive, dans le cadre de la formation dispensée par le Centre de formation régional aux métiers du patrimoine à Siem Reap (2008-2009).

La mission a été le fruit d'un travail d'équipe regroupant, outre l'Inrap, les archéologues, ingénieurs et architectes de l'Apsara, ainsi que les consultants et le chef du projet FSP, Michel Verrot, architecte des Bâtiments de France. Notons aussi que, pour chaque opération, nous avons pu bénéficier des informations avisées des chercheurs de l'Efeo travaillant à Siem Reap.

La demande des diverses institutions portait également sur la formation et l'accompagnement d'archéologues cambodgiens et des personnes ayant à charge la protection et la mise en valeur du patrimoine angkorien. Un document illustré accessible est en cours d'élaboration : il porte à la fois sur les notions indispensables à la compréhension des stratigraphies, sur des 